\title{
Electron cyclotron emission imaging diagnostic on TEXTOR
}

\author{
B. H. Deng, C. W. Domier, and N. C. Luhmann, Jr. \\ Department of Applied Science, University of California at Davis, Davis, California 95616
}

A. J. H. Donné and M. J. van de Pol

FOM-Instituut voor Plasmafysica Rijnhuizen, Associatie EURATOM-FOM, P.O. Box 1207, 3430 BE,

Nieuweigen, The Netherlands

(Presented on 19 June 2000)

\begin{abstract}
A 16-channel electron cyclotron emission imaging diagnostic system, developed by UC Davis in collaboration with the FOM-Instituut voor Plasmafysica Rijnhuizen, has been installed on the Torus Experiment for Technology Oriented Research tokamak. The system is designed to collect multichannel electron temperature profile and fluctuation data along a vertical chord of constant magnetic field, by collecting second harmonic $X$-mode emission. Technical details of the system are presented together with preliminary experimental data. (C) 2001 American Institute of Physics.
\end{abstract}

[DOI: $10.1063 / 1.1316748]$

\section{INTRODUCTION}

In magnetized plasmas such as that in tokamaks, electron cyclotron emission (ECE) arises from the gyro motion of electrons at harmonics of the cyclotron frequency, $\omega_{\mathrm{ce}}$. As the magnetic field strength of a tokamak depends on the major radius of the tokamak $(R)$, so does the ECE frequency

$$
\omega_{n}=n \omega_{\mathrm{ce}}=\frac{n e B(R)}{\gamma m_{e}}=\frac{n e}{\gamma m_{e}} \frac{B_{0} R_{0}}{R},
$$

where $n$ is the harmonic number, $\gamma$ is the relativistic factor, $R$ is the tokamak major radius, and $B_{0}$ is the magnetic field strength at the magnetic axis (at $R_{0}$ ) of the tokamak. ${ }^{1}$ In optically thick plasmas, the intensity of the ECE radiation is proportional to the local electron temperature $T_{e}$ :

$$
I_{n}=\frac{\omega^{2} T_{e}(R)}{8 \pi^{3} c^{2}} .
$$

Thus, information regarding the plasma electron temperature and its fluctuations can be obtained by measuring the intensity of optically thick ECE harmonics.

From Eq. (1), it is seen that temperature profiles can be obtained by resolving the spatially varying ECE frequency. Based on this, ECE radiometry has been a standard diagnostic for magnetic fusion plasmas for over 25 years. ${ }^{2}$ However, they are mostly limited to one-dimensional measurements along the major radius and generally have limited poloidal spatial resolution.

Recently, a novel plasma diagnostic, electron cyclotron emission imaging (ECEI), has been developed and operated on the Texas Experimental Tokamak Upgrade (TEXT-U) and Rijnhuizen Tokamak Project (RTP) tokamaks. ${ }^{3-5}$ The principle of the diagnostic is illustrated in Fig. 1. Instead of a single receiver horn as in a conventional ECE radiometer, the ECEI diagnostic utilizes newly developed millimeter wave imaging arrays as a multichannel receiver. These diagnostic systems have demonstrated excellent poloidal spatial resolution and two-dimensional measurement capability, and have proven to be an extremely useful tool for the study of fine scale structures of $T_{e}$ profiles and small amplitude plasma fluctuations. ${ }^{5-7}$

In continuation of this diagnostic development research, a 16-channel ECEI system has been developed and installed on the Torus Experiment for Technology Oriented Research (TEXTOR). It is based largely on the design of the previous RTP ECEI system, and reuses much of its optical and electronics hardware. Details of the system, including the constraints leading to the modified optical design, are presented in Sec. II. Preliminary plasma data are presented in Sec. III.

\section{THE TEXTOR ECEI SYSTEM}

The TEXTOR ECEI imaging optics were designed with two primary goals. First, it had to reuse as many of the previous RTP ECEI lenses and support framework as possible. Second, as was the case on RTP, it had to coexist on the same port as a double-pulse high spatial resolution Thomson scattering (TS) system, so that the ECEI system can be calibrated for $T_{e}$ profile measurements, and for comparison. Unlike the RTP design, in which the two diagnostics had a common beam path and were separated via a wire grid po-

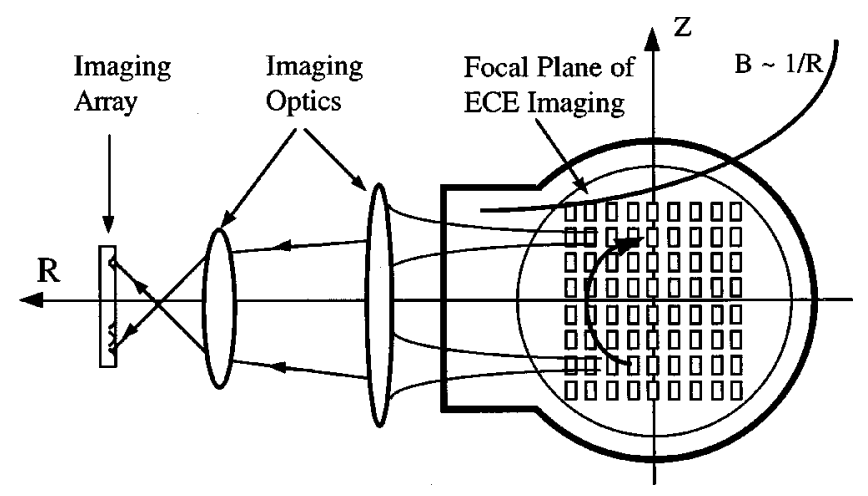

FIG. 1. Principle of the ECE imaging diagnostic. 


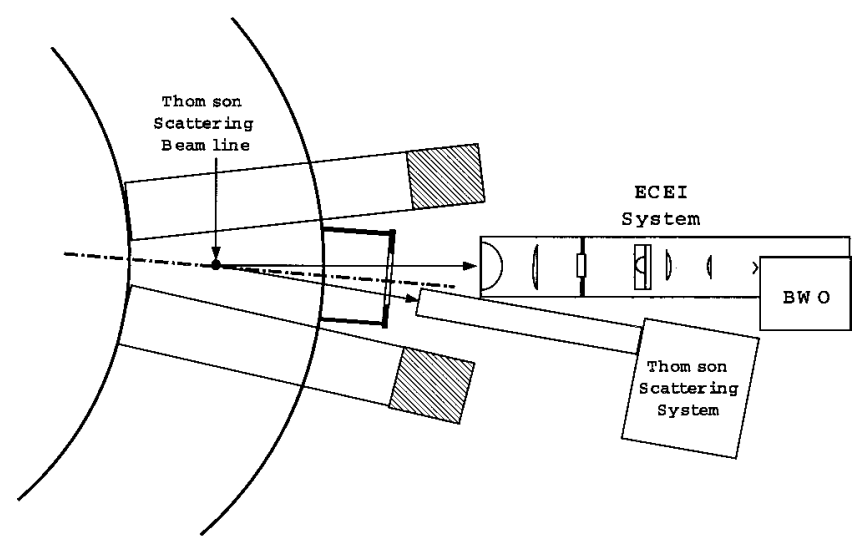

FIG. 2. Top view of the TEXTOR ECEI system.

larizer, the two systems in TEXTOR must sit side by side and therefore occupy only half of the available window area (45 $\mathrm{cm}$ high, $30 \mathrm{~cm}$ wide).

Figure 2 shows the top view of the TEXTOR ECEI and TS diagnostics. The default vertical viewing chord position of the ECEI system (defined by the most frequently employed plasma conditions and resultant local oscillation frequency) is designed to lie on top of the TS viewing chord. This simplifies the calibration of the ECEI system. The imaging lens closest to the window is the largest $(61 \mathrm{~cm}$ vertically, $28 \mathrm{~cm}$ toroidally, and the maximum thickness $13 \mathrm{~cm}$ ). However, the available window area for ECEI is only $15 \mathrm{~cm}$ wide. To avoid a physical conflict between the TS and ECEI systems and to avoid making new lenses and new support frames, the TS system is tilted by $6^{\circ}$ in the toroidal direction with respect to the normal of the window, and the center line of the ECEI system is tilted toroidally by $4.4^{\circ}$ in the opposite direction. Also, the ECEI system is pulled further back from the window. This positions the plasma-facing lens $146 \mathrm{~cm}$ away from the focal plane of the ECEI system, which coincides with the TS viewing chord. This distance is $20 \mathrm{~cm}$ further than that for the RTP ECEI system. Fortunately, we were able to accommodate the required changes by adjusting the relative positions of the array and various lenses.

The final design is shown in Fig. 3, which provides a side view of the ECEI system. This optical layout is different from that employed on RTP, as we are now able to place all optical components in a single line stretching from the

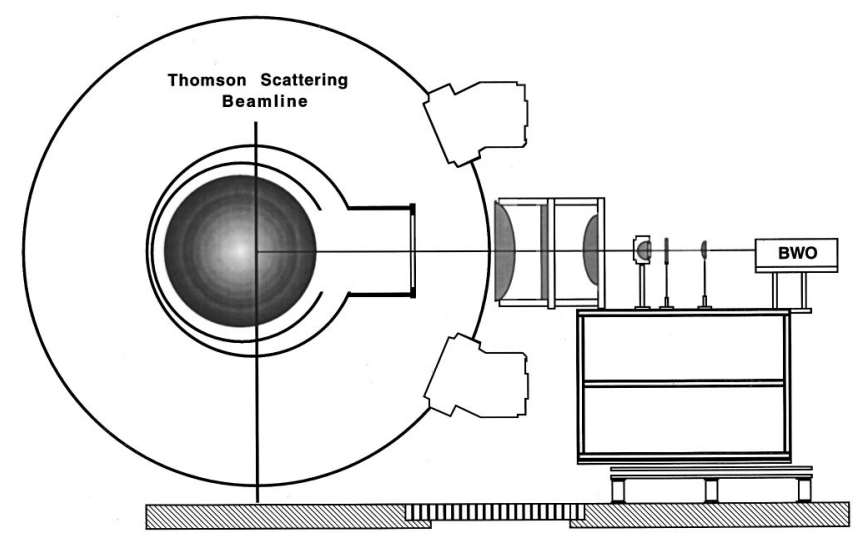

FIG. 3. Side view of the TEXTOR ECEI system.

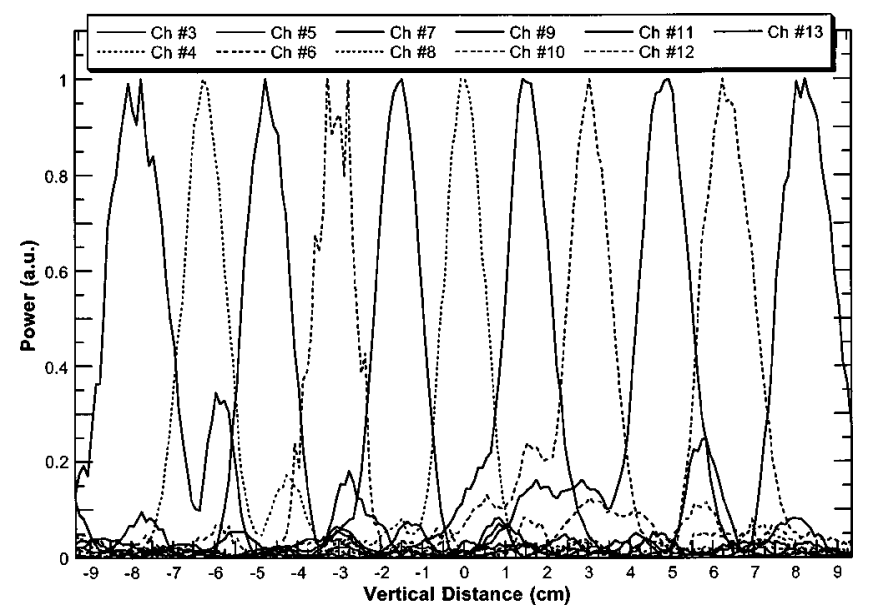

FIG. 4. E-plane focal plane beam patterns of the TEXTOR ECEI system.

plasma through the ECEI mixer array to the local oscillator. This was not possible in RTP or TEXT-U due to severe space constraints. The optical framework that supports the ECEI optics and electronics is reused with some modification.

Similar to the previous TEXT-U and RTP ECEI systems, the channel positions and focal plane beam patterns of the ECEI system on TEXTOR were characterized in the laboratory prior to installation on TEXTOR. Plotted in Fig. 4 are the $E$-plane (vertical direction) focal plane patterns measured at $118 \mathrm{GHz}$. It is seen that the beam spot sizes vary from $11 \mathrm{~mm}$ on the plasma midplane to about $18 \mathrm{~mm}$ for the outermost channels. The side lobes are primarily due to edge diffraction, and their effects on experimental measurements are discussed in detail in Ref. 8. The $H$-plane (toroidal direction) spot size is approximately $25 \mathrm{~mm}$. The $E$-plane interchannel spacing varies from $15 \mathrm{~mm}$ for the middle channels to $20 \mathrm{~mm}$ for the outermost channels. The $H$-plane spacing is $32 \mathrm{~mm}$. These parameters are close to that achieved in our previous ECEI systems.

Other than the changes discussed above, we were able to keep the electronics for the RTP ECEI system intact. In addition, the dichroic filter utilized on RTP to filter out 110

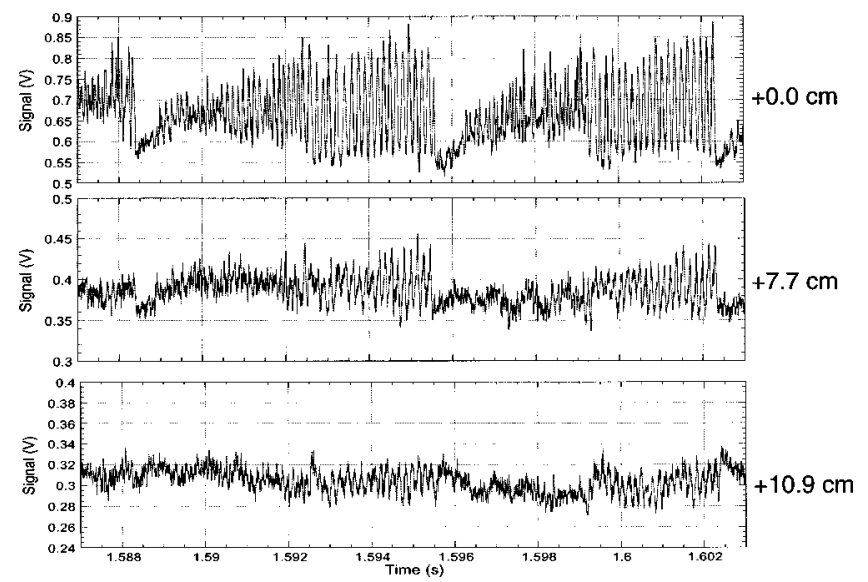

FIG. 5. ECEI data collected during $110 \mathrm{GHz}$ ECRH heating on TEXTOR with a toroidal field of $2.05 \mathrm{~T}$. The ECEI system was operated at $114.1 \mathrm{GHz}$, and the sample volumes are at $0 \mathrm{~cm}$ (top trace), $7.7 \mathrm{~cm}$ (middle), and 10.9 $\mathrm{cm}$ (bottom) above the plasma midplane. 
$\mathrm{GHz}$ stray radiation of the electron cyclotron resonance heating $(\mathrm{ECRH})$ system $^{3,5}$ is also reused on TEXTOR.

\section{INITIAL EXPERIMENTAL RESULTS}

Since its installation on TEXTOR in March, 2000, the ECEI system has been utilized to study a wide range of transport issues. Plotted in Fig. 5 are time traces from three ECEI channels during high power (400 kW) ECRH (shot 86875). The toroidal field is $2.05 \mathrm{~T}$, and the ECEI frequency is $114.1 \mathrm{GHz}$. The magnetic field is chosen so that the 110 $\mathrm{GHz}$ ECRH power is deposited near the Shafranov-shifted plasma center. The sample volumes of the ECEI system are located $6 \mathrm{~cm}$ apart from the ECRH resonance (to the high field side of the tokamak).

Two sawteeth cycles are shown in Fig. 5. Due to localized ECRH, a strong coherent oscillation with an amplitude equal to that of the sawteeth crashes $(\sim \pm 260 \mathrm{eV})$ is observed. These oscillations decrease in magnitude from the center $( \pm 20 \%$ relative amplitude) to the edge, with $\pm 10 \%$ and $\pm 5 \%$ at 7.7 and $10.9 \mathrm{~cm}$, respectively, above the midplane. This observation is very similar to that observed in TEXT-U and RTP, ${ }^{3,6}$ and is being analyzed further.

\section{ACKNOWLEDGMENTS}

This work is supported by the U.S. Department of Energy under Contract Nos. DE-FG03-95ER-54295 and DEFG03-99ER54531, and by NWO and EURATOM.

${ }^{1}$ I. H. Hutchinson, Principles of Plasma Diagnostics (Cambridge University Press, New York, 1987).

${ }^{2}$ A. E. Costley, R. J. Hastie, J. W. M. Paul, and J. Chamberlain, Phys. Rev. Lett. 33, 758 (1974)

${ }^{3}$ B. H. Deng, Ph.D. dissertation, UC Davis, 1999.

${ }^{4}$ R. P. Hsia, B. H. Deng, W. R. Geck, C. Liang, C. W. Domier, N. C. Luhmann, Jr., D. L. Brower, and G. Cima, Rev. Sci. Instrum. 68, 488 (1997).

${ }^{5}$ B. H. Deng, R. P. Hsia, C. W. Domier, S. R. Burns, T. R. Hillyer, N. C. Luhmann, Jr., T. Oyevaar, and A. J. H. Donné, Rev. Sci. Instrum. 70, 998 (1999).

${ }^{6}$ G. Cima, K. W. Gentle, A. Wootton, D. L. Brower, L. Zeng, B. H. Deng, C. W. Domier, and N. C. Luhmann, Jr., Plasma Phys. Controlled Fusion 40, 1149 (1998).

${ }^{7}$ B. H. Deng, D. L. Brower, G. Cima, C. W. Domier, N. C. Luhmann, Jr., and C. Watts, Phys. Plasmas 5, 4117 (1998).

${ }^{8}$ B. H. Deng, C. W. Domier, R. P. Hsia, N. C. Luhmann, Jr., D. L. Brower, G. Cima, A. J. H. Donné, T. Oyevaar, and M. J. van de Pol, Rev. Sci. Instrum. (these proceedings). 